\title{
Cell Automata Based Two Lanes Freeway Traffic Simulation
}

\author{
Run Zhang \\ School of Traffic and Transportation,Beijing jiaotong University,Beijing 100044, China. \\ 15120786@bjtu.edu.cn
}

Keywords: traffic flow simulation, cellular automata, Nagel-Schreckenberg Car Following, lane changing.

\begin{abstract}
The research goal of traffic flow theory is to set up a traffic flow model which can describe the actual traffic general characteristic, finding the basic rule of traffic flow. Due to the nature of the cellular automata, applying the cellular automata to the traffic flow has become a hot issue in the field of traffic flow. Cellular automata traffic flow model is established for simulation as a means to reveal the rule of traffic flow.In this paper, based on cellular automata model, we established two-lane traffic flow simulation model according to cars following rules of $\mathrm{Mr}$ Nagel and Schreckenberg and "safety-incentive" lane changing rules. And simulate the model on the multi-agent simulation platform Netlogo.
\end{abstract}

\section{Introduction}

Transportation system is an important part of human life, social and economic activities.A good transportation systems is the essential basic conditions of people's daily life,it can promote rapid economic development and the normal operation of enterprises. But, with the continuous development of national economy, people's demand for travel is also increasing, various traffic problems are springing up constantly, especially in Chinese cities. In recent years, in order to improve traffic conditions and ease the traffic congestion, many cities in China have made a lot of effort, but the traffic is still no obvious improvement. Investigate its reasons, are the lack of the guidance of advanced theory and the poor study of the internal evolution mechanism of traffic behavior and traffic flow. Therefore, in order to improve traffic conditions and alleviate the urban traffic congestion, the study of traffic flow theory is indispensable.

The goal of traffic flow theory research is to build an accurate and proper description on traffic characteristics of traffic flow model. According to different research methods, the communication model can be classified: macro continuous model, medium gas dynamic theory model, micro car following model (including cellular automata model) ${ }^{[1]}$.Cellular automata as a hot research topic in the field of traffic flow, has its unique advantages in terms of transportation.Cellular automata is discrete in time and space, when applied to traffic flow study, time and space variables can be selected for the discrete values. In essence, traffic elements is discrete, when use the cellular automata theory to study traffic flow, we can prevent the occurrence of discrete and continuous discrete process. At the same time, we can determine the rules of vehicle driving, as many samples as possible data to summarized the traffic following laws. When performed on computer, cellular automata model is more simple and practical, can amend its rules well according to different traffic conditions.

\section{Methodology}

In this section, we will introduce the traffic flow simulation model. The model contains two parts: the vehicle following rules and lane change rules.

\subsection{The Vehicle Following Rules}

In the vehicle following rules ${ }^{[2]}$,if the space between the vehicle and its front vehicle is greater than the vehicle current speed plus one and the vehicle current speed is less than the maximum speed, namely when the headway is equal to or greater than the vehicle current speed and the current speed is less than or equal to the maximum speed was founded at the same time, the vehicle 
speed increase for the vehicle current speed plus one. If the space between the vehicle and its front vehicle is less than the vehicle current speed, we can set the headway as the vehicle speed, namely when the headway is equal to or less than the vehicle current speed, vehicle speed is set to the headway.

In addition, two-lane model has stochastic slowing down rules.If the vehicle current speed is greater than zero and the probability of random moderated $\mathrm{p}$ is greater than or equal to zero is less than one, the vehicle speed reduced by one.

\subsection{Lane Change Rules}

In the lane changing rules ${ }^{[3]}$, we need to consider whether it conform to safety standards when want to change lane.Let us start from safety. Safety means that there are enough space in the front and behind of the vehicle which wants to change lanes and is on the target lane. Here we use [- $\mathrm{v}_{\max }$, $\mathrm{v}$ ] to describe safety standards (security criterion). Vmax is the maximum vehicle speed, $\mathrm{v}$ is the speed of the vehicle which wants to change lanes. That is to say, if a car want to change lane,the distance between the car and the vehicle in front of it on the target lane must satisfy that the distance must greater than or equal to the car speed; The distance between the car and the vehicle behind it on the target lane must satisfy that the distance must greater than or equal to the maximum speed.

After meeting the safety standards, we also should consider that if the lane changing cars have good reason s to change lanes, namely considering motivation standard (incentive criterion). In this article, we use the German standard to change lanes. In Germany, the lane on the right side must be used by default, and the vehicle must be on the left side.

First we consider the vehicle from the right lane to the left lane: in accordance with the standards of Germany, when the vehicle in the right lane,overtaking is not allowed.If there is a slow car on the left lane, and another car want to change to the left lane from the right lane, it need to after the slow speed. Therefore, if a car want to move to the left lane, there need to have a car on the left lane or right lane less than or equal to the speed of the vehicle which want to change lanes.And if a car want to move to the right lane,the speed of this car must satisfy that it is less than the speed of vehicles on the left lane or on the right lane.

\section{The simulation results}

After the two-lane simulation, we need to record some data for the analysis of simulation results. Netlogo provides us with the function of the output data, we can record some data which can better analyze the simulation results. Netlogo has two main ways to deal with the files: to read and write. The difference between the two way is that whether the data is go into the platform and used by the platform or go out from the platform for the user. Reading refers to the data read from the file, used in the model; Writing refers to the data from the model output to a file. Writing has been applied in our simulation, the simulation data output to excel file.

Qualitative and quantitative characteristics of traffic flow running state is one of the characteristics of traffic flow,traffic flow parameters is used to describe characteristic quantities of traffic flow, and the changing rule of the traffic flow parameters reflect the basic characteristics of traffic flow.In the simulation, we need to record the related numerical traffic flow parameters, such as the number of cars, car distance, average speed, etc. These are obtained constantly.we can write program to output the results to excel,and deal with the data in excel to get some conclusions. 


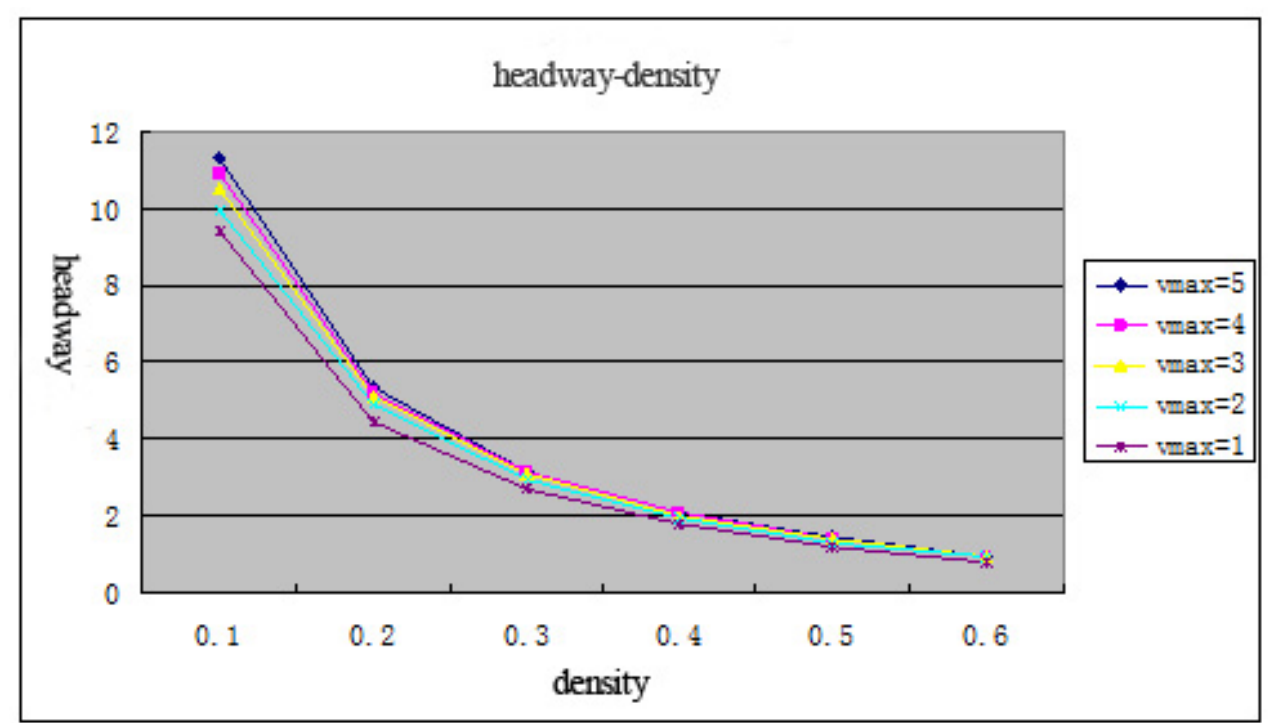

Fig. 1The curves of headway changing

Figure 1 shows when the random rate of slowing down is fixed, $v_{\max }=1,2,3,4,5$, the headway changes with density. We can see from the chart, when the density is fixed,the greater the maximum speed is, the greater the headway is; With the increase of density, line is more and more gentle. With the increase of density, the headway gradually tends to zero. This means that, on the road, if the traffic density is fixed, the maximum speed of the vehicle is allowed to larger.No matter what lane vehicle performance, if the density is too large, it will cause traffic jams.

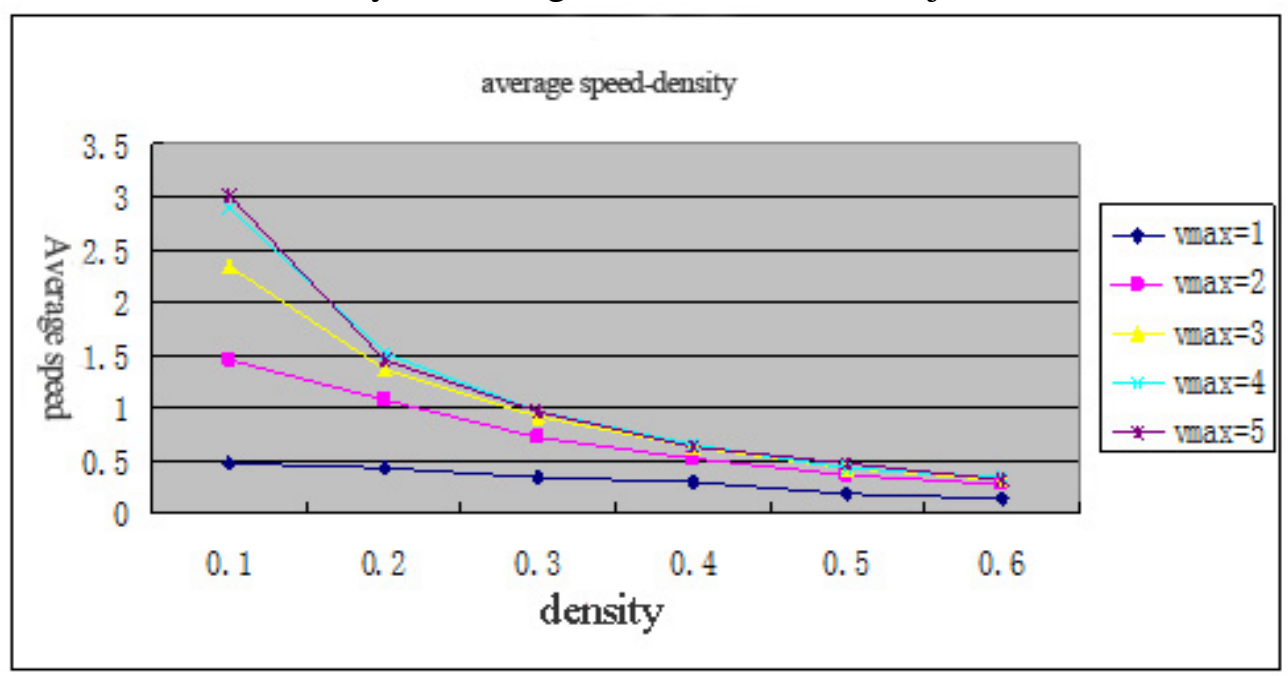

Fig. 2 The curve of average speed and density

Figure 2 shows when the random rate of slowing down is fixed, $v_{\max }=1,2,3,4,5$, the average speed change changes with density. By the graph, we can see that under a certain density, the greater the maximum velocity, the greater the average speed; when maximum speed is the same, average velocity decreases with the increase of the density, and with the increase of density, line is more and more smooth.With the increase of density, the average speed tends to zero. When the density is too large, regardless of maximum speed, will cause traffic jams.

\section{Conclusion}

Based on cellular automata traffic flow simulation model is designed and implemented by many aspects of the theory, methods and techniques, this model also has a lot of problems to be solved and perfected.In establishing a two-lane model based on cellular automata, we should as far as possible close to the actual traffic situation,and study further to establish a model which is suitable for all kinds of road traffic flow based on cellular automata. 


\section{References}

[1] Jia Bin,Gao Ziyou,Li Keping, et al. Traffic system modeling and simulation based on cellular automata. Beijing: Science Press, 2007.

[2] K. Nagel, M. Schreckenberg. A Cellular automaton model for free way traffic. J.Phys. I(France),1992,2:2221-2229.

[3] K. Nagel, D.E. Wolf, P. Wagner, et al. Two-lane traffic rules for cellular automata: A systematic approach. Physical Review E1998,58(2):1425-14. 